\title{
Avoiding dislocations in ion-implanted silicon
}

\author{
F.W. Saris ${ }^{1}$, J.S. Custer ${ }^{1}$, R.J. Schreutelkamp ${ }^{1,2}$. \\ R.J. Lieftingl,3, R. Wijburg 3 , H. Wallinga ${ }^{3}$.
}

1) Fom Institute for Atomic and Molecular Physics, Kruislaan 407, 1098 SJ Amsterdam, the Netherlands

2) IMEC vzw, Kapeldreef 75, B-3001 Leuven, Belgium

3) University of Twente, MESA Research Institute, P.O. Box 217, 7500 AE Enschede, The Netherlands

\begin{abstract}
Damage from ion implantation in Si can lead to dislocation formation during subsequent thermal annealing. These dislocations may sharply degrade device performance, making it desireable to suppress their formation. In this paper the criterion for dislocation formation is reviewed. Knowing this criterion suggests several ways to avoid dislocation formation in high dose implants for device applications.
\end{abstract}

\section{INTRODUCTION}

For future submicron devices one should be able to use high energy ion implantation for tailoring of doping profiles. It will then be necessary to anneal the ion implantation damage such that no dislocations or other secundary defects are present in the active areas of the device. Therefore secondary defect formation after ion implantation has become an issue again. In recent years various suggestions have been made to explain the formation of dislocations during annealing of primary damage, made by ion implantation below the critical dose for amorphization.

It has been suggested that dislocation formation depends on: 1) the implanted ion species, 2) the number of excess atoms introduced during implantation, 3) a critical impurity dose, 4) a critical impurity concentration and 5) a critical peak concentration of displaced Si atoms. Below it will be shown that neither of these suggestions are consistent with the experimental findings. Instead, dislocation formation appears to depend critically on the total number of $\mathrm{Si}$ atoms displaced during implantation. Once the criterion for dislocation formation is known, it is also understood how dislocations may be avoided. This will be the message of our paper. 


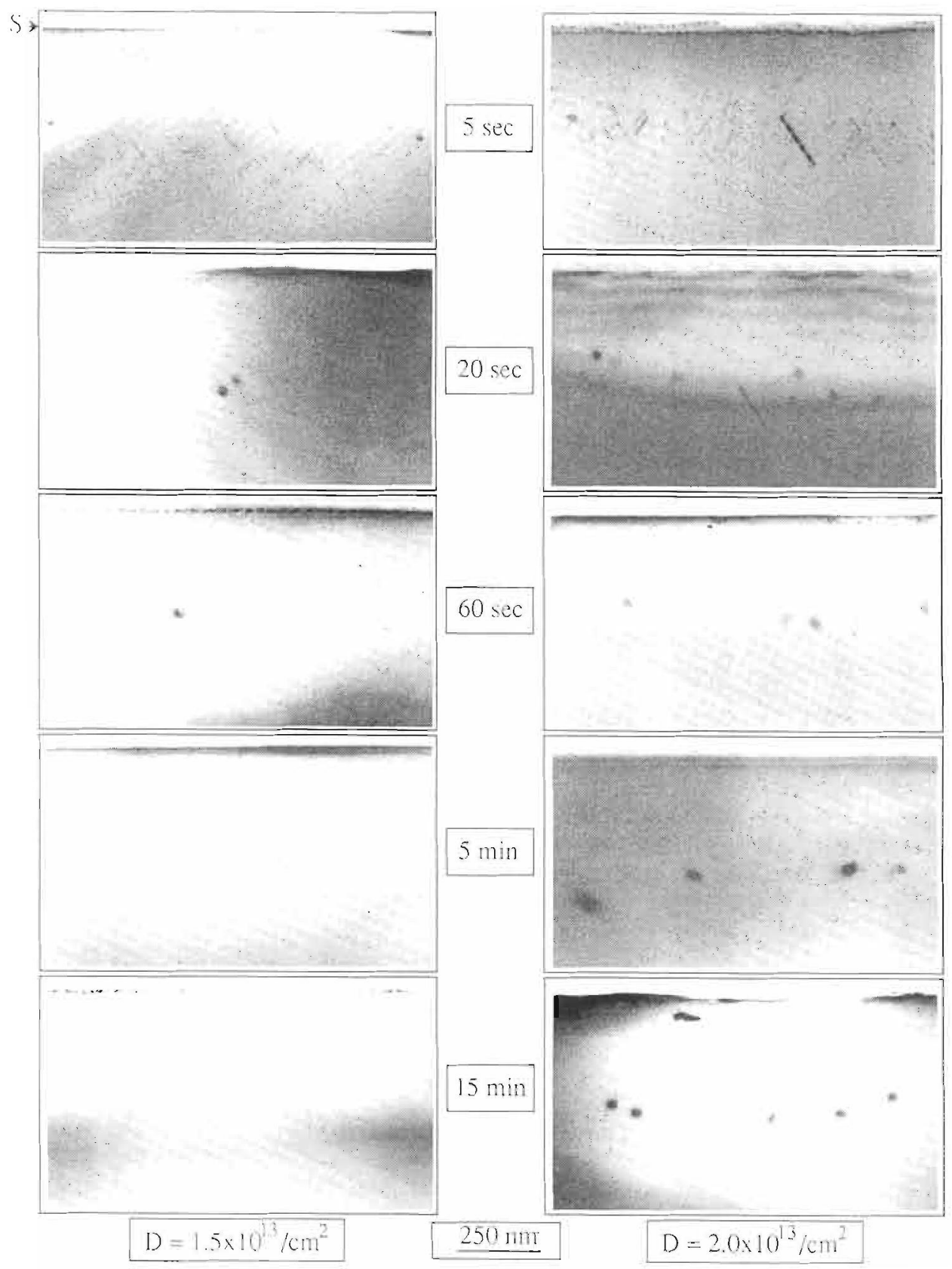

Fig. 1: XTEM micrographs after $900{ }^{\circ} \mathrm{C}$ annealing of Si implanted with $1 \mathrm{MeV}$ In to doses of 1.5 or $2.0 \times 10^{13 /} \mathrm{cm}^{2}$. Anneal times are indicated in the figure; from ref. 1 . 


\section{CRITERION FOR THE FORMATION OF DISLOCATIONS IN ION-IMPLANTED SILICON.}

First, let us look at an example of silicon implanted with $1 \mathrm{MeV}$ In to doses of 1.5 and 2.0 $x 10^{13} / \mathrm{cm}^{2}$. The samples were annealed at $900^{\circ} \mathrm{C}$ to remove the primary damage. Anneals for $5,20,60$ and $300 \mathrm{sec}$ were performed in a rapid thermal annealer (RTA) while a $1.5 \mathrm{~min}$. anneal was performed in a vacuum furnace.

Fig. 1 shows a cross section transmission electron miscroscopy (XTEM) study of the recovery of the damage as a function of the anneal time. After the $5 \mathrm{sec}$ anneal, both doses result in the formation of a very high concentration, $\sim 10^{11} / \mathrm{cm}^{2}$, of dislocation rods. There rods are only $10 \mathrm{~nm}$, and represent an early stage in the dislocation formation process. Larger rods and some small dislocation loops are also visible in the micrographs. After $20 \mathrm{sec}$, a large difference is observed, with the low dose sample containing only a few loops whereas this number is much higher for $2.0 \times 10^{13} \mathrm{In} / \mathrm{cm}^{2}$. If the anneal is done for $60 \mathrm{sec}$, for the low In dose only one dislocation is observed, whereas the sample with the high dose is essentially unchanged compared to the $20 \mathrm{sec}$ anneal. Clearly, a stable defect configuration is obtained for an In dose of $2.0 \times 10^{13} / \mathrm{cm}^{2}$, whereas the dislocations formed for $1.5 \times 10^{13} \mathrm{In} / \mathrm{cm}^{2}$ are too small to be stable at $900^{\circ} \mathrm{C}$. This is confirmed if the anneal time is prolonged to 5 or $15 \mathrm{~min}$. No dislocations are observed in the low dose sample, while they remain for the high dose.

The principal of the criterion for dislocation formation is clearly demonstrated in this example. Primary-damage amounts, differing by only 30\%, were generated by In implants. For short anneal times roughly the same type of dislocation rods are observed for both implants. The number of rods is higher for $2 \times 10^{13} \mathrm{In} / \mathrm{cm}^{2}$. However, these rods are an early stage in the dislocation formation process and are not stable. Some of the rods will grow on the expense of others and will turn into loops. Loops smaller than a critical size are not stable at $900^{0} \mathrm{C}$ and will disappear if the anneal is continued. This is the case for $1.5 \times 10^{13} \mathrm{In} / \mathrm{cm}^{2}$, whereas somewhat larger loops have formed for the $210^{13} \mathrm{In} / \mathrm{cm}^{2}$ implant. Therefore, the total number of silicon atoms displaced by the implant forms the critical parameter.

We have demonstrated ${ }^{2)}$ for a wide range of ion energies and ion masses that the criterion for dislocation formation is the number of displaced atoms $/ \mathrm{cm}^{2}$. These displaced atoms provide the mobile $\mathrm{Si}$ interstitials which agglomerate to form dislocations. The critical number of displaced atoms depends on the ion mass, increasing from $10^{16} \mathrm{Si} / \mathrm{cm}^{2}$ for $\mathrm{B}$ to $10^{17} \mathrm{Si} / \mathrm{cm}^{2}$ for Sb. (see fig. 2). 


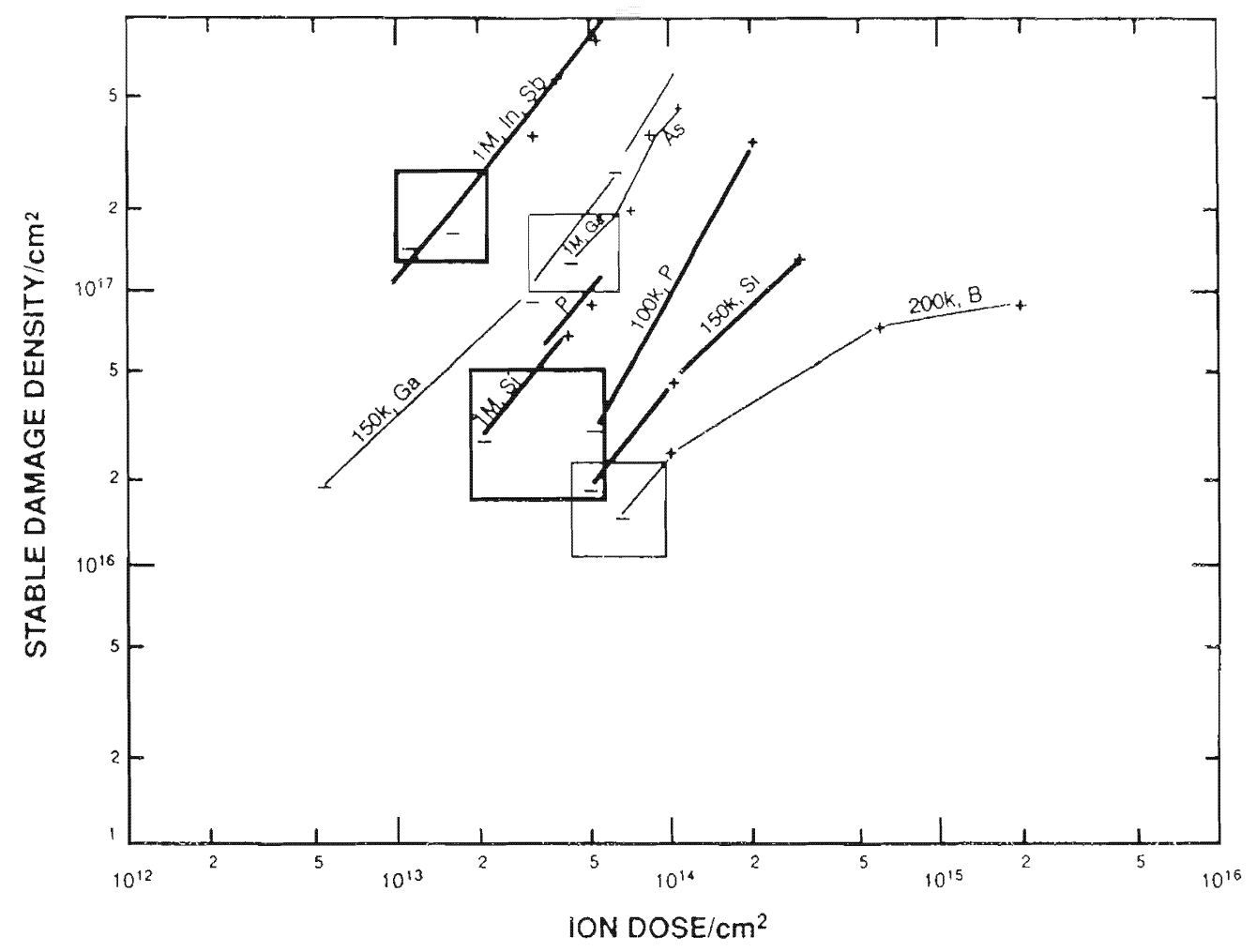

Fig. 2.: Stable damage density plotted against ion dose for different mass ions and energies.(Energies are denoted as $\mathrm{k}(\mathrm{keV})$ or $\mathrm{M}(\mathrm{MeV})$; + data are dislocations formed after $900^{\circ} \mathrm{C}$ for 15 minutes anneal, - data are where no dislocations formed. Squares represent a transition region; from ref. 2 and 3.

\section{ENGINEERING OF ION IMPLANTATION DAMAGE}

Since it is now known under what conditions dislocations form during annealing of ion implantation damage, one should also know how to avoid them or how to nucleate dislocations in an area of the silicon wafer where they do not affect the electrical properties.

First, it should be pointed out that channeling implantations are advantageous over random implants as far as the formation of dislocations is concerned. For the same ion dose, during an implant in the channeling direction fewer silicon atoms will be displaced, hence for channeling implants dislocation formation is observed at much higher doses than for random implants 2 ).

Multiple implantations and annealing is yet another way of increasing the ion dose and still avoiding dislocation formation. Knowing that a certain amount of damage is required to form dislocations suggests to perform the implant in several subcritical steps, annealing out the damage between each step. This has been demonstrated for the In implant as well as for the common dopants B, P and As, as is illustrated in Fig. 3. 


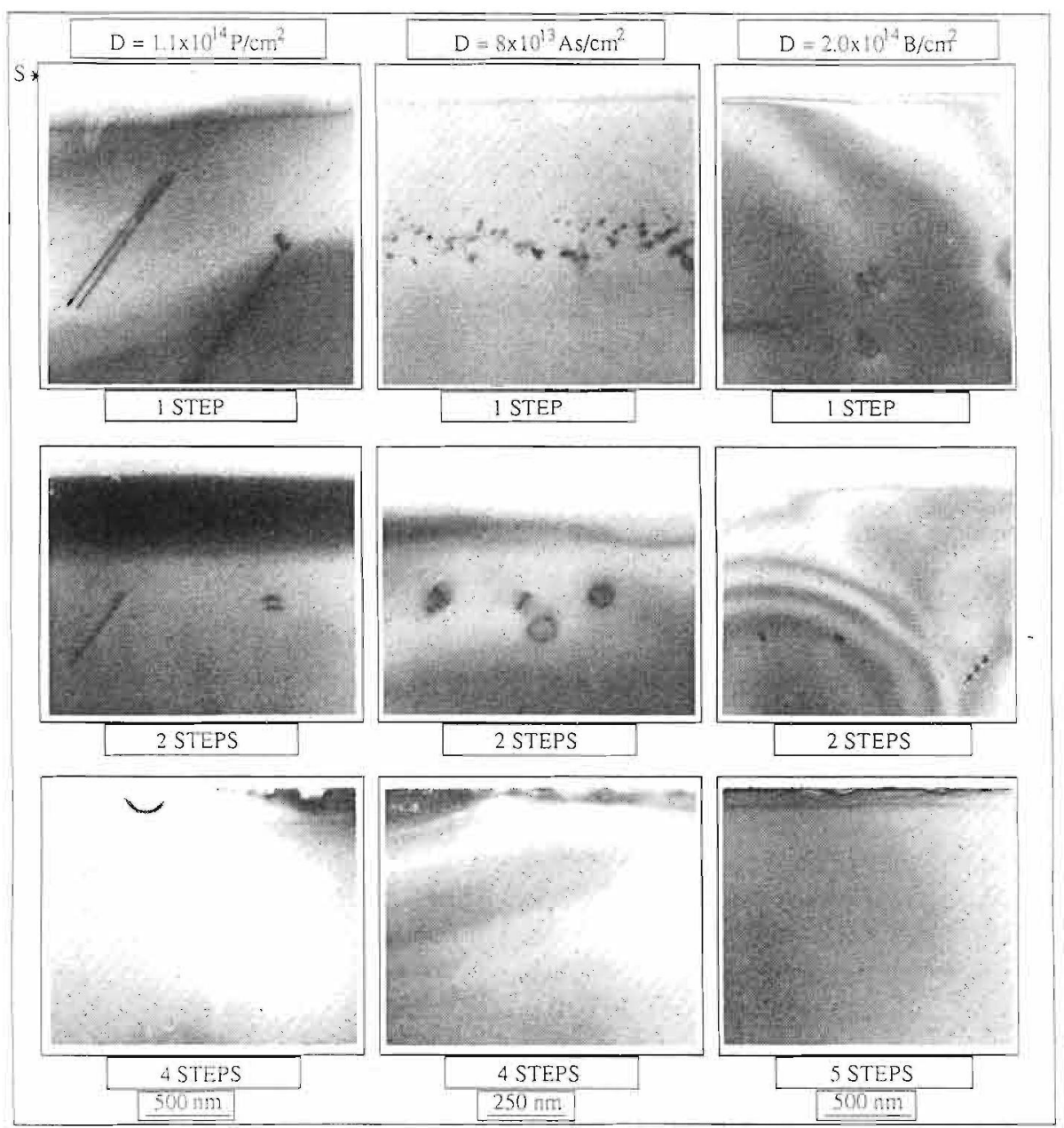

Fig. 3. XTEM analysis of Si implanted with $1 \mathrm{MeV} 1.1 \times 10^{14} \mathrm{P} / \mathrm{cm}^{2}$. The number of implant and anneal steps used are denoted in the Figure, from ref. 1.

A third method for suppressing dislocation formation relies on the curious behavior of carbon implants, which do not lead to dislocation formation even for damage levels for above what is required for boron. This is attributed to carbon atoms being able to getter some number of $\mathrm{Si}$ interstitials. We have demonstrated that implanting carbon over a dopant implant can suppress dislocation formation 1). 


\section{CONCLUSIONS FOR DEVICE APPLICATIONS}

The above engineering schemes have been applied to a $4 \times 10^{13} / \mathrm{cm}^{2} 1.5 \mathrm{MeV} \mathrm{P}$ implant used to create the collector in a vertical bipolar transistor process. In these devices, dislocations may increase the leakage current and even lead to collector - emitter shorts. These shorts reduce the transistor yield. The use of multiple step implants or the introduction of a $\mathrm{C}$ gettering layer have been demonstrated to avoid dislocation formation. Recent experimental results show that these schemes successfully lower the leakage current and dramatically increase device yield 1,4 ). The presence of $\mathrm{C}$ can cause an increased collector/substrate leakage, indicating that the $\mathrm{C}$ profile needs to be optimized with respect to the doping profiles.

Another implication of a critical amount of damage is that restricting the size of the implanted area should help suppress dislocation formation by, for example, increasing the outdiffusion of point-defects from the damaged region. Implanting into sub-micron cricles drastically lowers the dislocation density compared with lines of a similar width. These examples show that an understanding of the parameters leading to dislocation formation from ion implant damage results in ways to avoid their formation and thereby increase device yield.

\section{ACKNOWLEDGEMENTS}

This work is part of the research program of the Stichting voor Fundamenteel Onderzoek der Materie (FOM) with financial support from the Nederlandse Organisatie voor Wetenschappelijk Onderzoek (NWO) and Varian Ion Implant Systems (Gloucester, MA, U.S.A.).

\section{REFERENCES}

1) J.R. Liefting, $\mathrm{PhD}$ thesis University of Twente, 1992

2) R.J. Schreutelkamp et al. Mater. Sci. Rep. 6 (1991) 275

3) T.E. Seidel and L.A. Larson, MRS Bulletin June 1992 p. 34

4) R.C.M. Wijburg et al. Proceedings of this meeting. 